\title{
ON MODULAR REPRESENTATION ALGEBRAS AND A CLASS OF MATRIX ALGEBRAS
}

\author{
J-C. RENAUD \\ (Received 12 January, 1981) \\ Communicated by D. E. Taylor
}

\begin{abstract}
Let $G$ be a cyclic group of prime order $p$ and $K$ a field of characteristic $p$. The set of classes of isomorphic indecomposable $(K, G)$-modules forms a basis over the complex field for an algebra $Q_{p}$ (Green, 1962) with addition and multiplication being derived from direct sum and tensor product operations.

Algebras $Q_{n}$ with similar properties can be defined for all $n \geqslant 2$. Each such algebra is isomorphic to a matrix algebra $\Re_{n}$ of $n \times n$ matrices with complex entries and standard operations. The characters of elements of $Q_{n}$ are the eigenvalues of the corresponding matrices in $\mathfrak{R}_{n}$.
\end{abstract}

1980 Mathematics subject classification (Amer. Math. Soc.): 20 C 20.

\section{Introduction}

Let $G$ be a cyclic group of prime order $p$ and $K$ a field of characteristic $p$. A $G$-module is a $(K, G)$-module with the elements of $G$ acting as right operators: there exist exactly $p$ distinct isomorphism classes of indecomposable $G$-modules, with $K$-dimension $1, \ldots, p$. (For further details, see Green, 1962 or Renaud, 1979.)

Choose representatives $V_{1}, \ldots, V_{p}$ from the classes with $V_{i}$ having $K$-dimension $i$. The modular representation algebra $\Theta_{p}$ has basis $\left\{V_{1}, \ldots, V_{p}\right\}$ over the complex field, with products defined by

$$
V_{r} \times V_{s}=\sum_{i=1}^{p} a_{i r s} V_{i}
$$

where $a_{i r s}$ is the number of modules isomorphic to $V_{i}$ in the direct sum decomposition of $V_{r} \otimes_{K} V_{s}$.

Copyright Australian Mathematical Society 1982 
This commutative product is expressible by the formula (Renaud, 1979):

For $1 \leqslant r \leqslant s \leqslant p$,

$$
V_{r} \times V_{s}=\sum_{i=1}^{c} V_{s-r+2 i-1}+(r-c) V_{p}
$$

where

$$
c= \begin{cases}r & \text { if } r+s \leqslant p, \\ p-s & \text { if } r+s \geqslant p .\end{cases}
$$

$Q_{p}$ may be regarded as generated by $V_{2}$ with relation $V_{r}=V_{2} \times V_{r-1}-V_{r-2}$ for $2<r \leqslant p$, restricted by $V_{2} \times V_{p}=2 V_{p}$, and hence elements in $\mathbb{Q}_{p}$ are polynomials in $V_{2}$.

This class of algebras can be extended in a natural way: for all integers $n \geqslant 2$ let $Q_{n}$ be the algebra with identity $V_{1}$ and generator $V_{2}$, defining relation for $V_{r}$ and restriction as above, with $p$ replaced by $n$. Note that this is not in general a representation algebra of a group: the $V_{i}$ are abstract elements, not modules.

We wish to show $\mathfrak{Q}_{n}$ is isomorphic to a particular matrix algebra $\Re_{n}$.

\section{The matrix algebra}

Let $\mathscr{T}_{n}$ be the algebra generated by the $n \times n$ matrix $W_{2}^{n}$ which has entries 1 on the sub- and super-diagonals, 1 at each end of the main diagonal, and 0 elsewhere. We wish to show $\mathfrak{R}_{n}$ is isomorphic to $\mathbb{Q}_{n}$.

Let $W_{1}^{n}$ be the unit $n \times n$ matrix. Let $W_{r}^{n}=W_{2}^{n} \times W_{r-1}^{n}-W_{r-2}^{n}$. Clearly necessary and sufficient conditions for the isomorphism to hold are that the restriction $W_{2}^{n} \times W_{n}^{n}=2 W_{n}^{n}$ hold, and that the $W_{r}^{n}$ be linearly independent. To show this, and also to describe the $W_{r}^{n}$ in general, we have

Proposition. $W_{r}^{n}$ is the $n \times n$ matrix $\left(a_{i j}\right)$ with

$$
\begin{array}{ll}
a_{i j}=1 & \text { if }(\mathrm{i}) i+j-1 \leqslant r \\
& \text { or (ii) } 2 n-(i+j-1) \leqslant r \\
& \text { or (iii) }|i-j|-1 \equiv r(\bmod 2) \text { and }|i-j|<r, \\
a_{i j}=0 & \text { otherwise. }
\end{array}
$$

Proof. The proposition clearly holds for $r=1$ and $r=2$. Examination of the pattern in diagrams of $W_{k}^{n} \times W_{2}^{n}$ versus those of $W_{k+1}^{n}$ and $W_{k-1}^{n}$ is then sufficient. 
COROLlaRY. (i) $W_{n}^{n}$ is the $n \times n$ matrix with every entry 1 . Hence $W_{2}^{n} \times W_{n}^{n}=$ $2 W_{n}^{n}$.

(ii) Examination of the first row of the $W_{r}^{n}$ shows they are linearly independent. Hence $\mathfrak{T}_{n}$ is isomorphic to $\mathbb{Q}_{n}$.

\section{The characters of $\mathbb{Q}_{n}$}

A character of $\mathbb{Q}_{n}$ is a non-trivial homomorphism, $\phi: \mathbb{Q}_{n} \rightarrow \mathbf{C}$ where $\mathbf{C}$ is the complex field. Green (1962) derived the characters for $Q_{p}$ : the general case is similar.

Let $x$ be indeterminate over a commutative, associative algebra with identity $i$. The Chebyshev polynomials $S_{k}$ are defined by

$$
S_{0}(x)=i, \quad S_{1}(x)=x, \quad S_{k}(x)=x S_{k-1}(x)-S_{k-2}(x) \quad \text { for } k \geqslant 2 .
$$

(For further details, see Abramovitz and Stegun (1972), Chapter 22.)

Now for $1 \leqslant r \leqslant n, V_{r}=S_{r-1}\left(V_{2}\right)$. Let $\phi: \mathbb{Q}_{n} \rightarrow \mathbf{C}$ be a character of $\mathscr{Q}_{n}$ : clearly $\phi\left(V_{1}\right)=1$ and since $V_{n} \times\left(V_{2}-2 V_{1}\right)=0, \phi\left(V_{n}\right)=0$ or $\phi\left(V_{2}\right)=2$. The second case gives the dimension character

$$
\delta\left(V_{r}\right)=r, \quad 1 \leqslant r \leqslant n .
$$

For the first case, let $\phi\left(V_{2}\right)=x$. Then we require $S_{n-1}(x)=0$, and this has solutions $x_{j}=2 \cos (\pi j / n), j=1, \ldots, n-1$.

Moreover, $S_{r-1}(2 \cos \theta)=\sin r \theta / \sin \theta, r \geqslant 1$, and hence there are $n-1$ other characters,

$$
\phi_{j}\left(V_{r}\right)=\frac{\sin (\pi j r / n)}{\sin (\pi j / n)}, \quad 1 \leqslant r \leqslant n, 1 \leqslant j \leqslant n-1 .
$$

\section{The eigenvalues of matrices in $\mathfrak{R}_{n}$}

The characteristic polynomial of $W_{2}^{n}$ is derived from the equation

$$
W_{n}^{n}\left(W_{2}^{n}-2 W_{1}^{n}\right)=0 \text {; }
$$

thus the eigenvalues of $W_{2}^{n}$ are the solutions of $S_{n-1}(\lambda)(\lambda-2)=0$, and these are just the characters at $V_{2}$. Hence the eigenvalues of $W_{r}^{n}$ are the character values at $V_{r}$. 
REMARK. This gives rise to an eigenvalue result of some minor interest: let $A$ be the $n \times n$ matrix symmetric about both diagonals, with

$$
a_{i j}=(-1)^{i+1}\left\{x_{j}+\sum_{k=1}^{i-1}(-1)^{k}\left(x_{j-k}+x_{j+k}\right)\right\}
$$

for $1 \leqslant i \leqslant \frac{1}{2}(n+1), i \leqslant j \leqslant n+1-i$, where $x_{j} \in \mathbf{C}, j=1, \ldots, n$.

For example, with $n=5$,

$$
\left(\begin{array}{ccccc}
x_{1} & x_{2} & x_{3} & x_{4} & x_{5} \\
x_{2} & x_{1}-x_{2}+x_{3} & x_{2}-x_{3}+x_{4} & x_{3}-x_{4}+x_{5} & x_{4} \\
x_{3} & x_{2}-x_{3}+x_{4} & x_{1}-x_{2}+x_{3}-x_{4}+x_{5} & x_{2}-x_{3}+x_{4} & x_{3} \\
x_{4} & x_{3}-x_{4}+x_{5} & x_{2}-x_{3}+x_{4} & x_{1}-x_{2}+x_{3} & x_{2} \\
x_{5} & x_{4} & x_{3} & x_{2} & x_{1}
\end{array}\right) .
$$

Now

$$
A=x_{n} W_{n}^{n}+\sum_{i=1}^{n-1}\left(x_{i}-x_{i+1}\right) W_{i}^{n}
$$

and hence the eigenvalues of $A$ are

$$
\lambda_{0}=\sum_{i=1}^{n} x_{i} \text { and } \lambda_{j}=\sum_{i=1}^{n-1}\left(x_{i}-x_{i+1}\right) \frac{\sin (\pi i j / n)}{\sin (\pi j / n)}
$$

for $j=1, \ldots, n-1$.

\section{The eigenvectors of $W_{r}^{n}$}

The eigenvectors of $W_{r}^{n}$ also have interesting properties. For $W_{2}^{n}$, the eigenvalues are $\lambda_{j}=2 \cos (\pi j / n), j=0, \ldots, n-1$. Let $\lambda_{j}$ have a corresponding eigenvector $\left[y_{1}, \ldots, y_{n}\right]$. It is not difficult to show that we may choose $y_{1}=1$, and that for $1<i \leqslant n$

$$
\begin{aligned}
y_{i} & =S_{i-1}\left(\lambda_{j}\right)-S_{i-2}\left(\lambda_{j}\right) \\
& =\cos (\pi i j / n)+\sin (\pi i j / n) \tan (\pi j / n) .
\end{aligned}
$$

Moreover, it can be deduced that this set of eigenvectors is orthogonal.

REMARK. Any matrix similar to $W_{2}^{n}$ will generate an algebra similar to on ${ }_{n}$ : one such with a pattern as clear as that of $W_{2}^{n}$ is the $n \times n$ matrix $\left(r_{i j}\right)$ with

$$
r_{i j}= \begin{cases}1 & \text { if }|i-j|=1, \quad i+j<2(n-1) \\ 2 & \text { if } i=j=n \\ 0 & \text { otherwise. }\end{cases}
$$


Of course, it is trivially obvious that the matrix $\bigoplus_{i=0}^{n-1}[2 \cos (\pi i / n)]$ also generates an algebra isomorphic to $\mathfrak{K}_{n}$.

\section{References}

M. Abramovitz and I. Stegun (1972). Handhook of mathematical functions (Dover, New York).

J. A. Green (1962), 'The modular representation algebra of a finite group', Illinois J. Math. 6 , 607-619.

J-C. Renaud (1979), 'The decomposition of products in the modular representation ring of a cyclic group of prime power order', J. Algebra 58 (1), 1-11.

Department of Mathematics

University of Papua New Guinea

Papua New Guinea 